SHORT REPORT

\title{
Role of anterior and occipital white matter lesions for smooth eye tracking in myotonic dystrophy
}

\author{
H Kimmig, M Petrick, M Orszagh, T Mergner
}

J Neurol Neurosurg Psychiatry 2002;72:808-81 1

Background: The degradation of smooth pursuit eye movements (SP) in myotonic dystrophy (MD) has been shown to result from lesions in the central nervous system. Imaging and histopathological studies have shown considerable areas of white matter lesions (WMLs) with periventricular emphasis.

Objective: To discover which of these WMLs are relevant in SP impairment?

Method: Horizontal sinusoidal SP, the vestibulo-ocular reflex (VOR), and its suppression by fixation (VOR-S) were investigated in 12 patients with $M D$, and the data obtained were compared with those from a control group. Results: Parallel degradation of SP and VOR-S was found in patients, although the eyes hardly needed to move with VOR-S. VOR in patients was normal. These results indicate a central rather than a peripheral origin for the SP degradation. Magnetic resonance images of the patients' heads were obtained and the WMLs transferred to a standard map. The lesions were mainly located around the occipital and anterior horn of the lateral ventricle. SP performance was then related to lesion site. The largest area of deficit associated lesions appeared to be in the parieto-occipital white matter. The most severe SP impairment, however, was associated with frontal WMLs.

Conclusion: The study establishes a link between SP deficits and WMLs in patients with MD, in line with previous observations that, not only parieto-occipital regions, but also the frontal cortex has a crucial role in the gain control of SP.

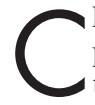
lear vision of a moving target is achieved by smooth pursuit eye movements (SP), which keep the target on the fovea. The SP system involves the striate cortex, the parieto-occipital and posterior parietal cortex, the frontal eye fields, dorsolateral pontine nuclei, cerebellum, vestibular nuclei, and oculomotor nuclei. ${ }^{1}$ SP impairment has been reported for cortical and adjacent white matter lesions (WMLs) in both frontal eye fields and parieto-occipital cortex. $^{23}$

SP has been shown to be impaired in myotonic dystrophy (MD), an autosomal dominant, multisystem disorder, which primarily involves muscles (myotonia, dystrophy), but other systems such as the central nervous system (CNS) as well. In a previous study, we showed that SP degradation results from CNS dysfunction rather than peripheral muscle deficits. ${ }^{45}$ It is worth mentioning that some authors did not find SP deficits in patients with $\mathrm{MD}^{6}{ }^{67}$ We attribute this to methodological differences, especially the low target velocities used in the latter studies (as discussed in ${ }^{8}$ ). Magnetic resonance imaging (MRI) in patients with MD has shown WMLs in the cerebral hemispheres. ${ }^{90}$ We decided to investigate to what extent the WMLs relate to the SP deficits in the patients and what the topological characteristics of this relation are. For this purpose, we obtained MRI scans of the patients' heads, standardised the extent of the WMLs, and correlated these with SP performance.

\section{METHODS \\ Subjects}

We examined 12 patients with MD (mean (SD) age, 47 (14) years) and 12 healthy control subjects (40 (15) years). The diagnosis of MD was based on the clinical examination, electromyographic recordings, and genetic analysis (all patients showing more than 100 CTG repeats ${ }^{11}$; mean age at clinical disease onset, 27 (16) years; visual acuity $>0.6$ ). MD severity was assessed by the Karnofsky index ${ }^{12}$ which is a global disability scale (mean Karnofsky index score 71 (12)\%; patients with this score were generally unable to carry out active work, but were able to take care of themselves). Routine clinical examinations of eye movements were normal. There were no indications of relevant additional diseases in the patients at the time of investigation. None of the patients or the controls were taking any drugs at the time of the measurements.

\section{Oculomotor stimuli and recording of eye movements} Stimulation and recording techniques were as in our previous study. ${ }^{4}$ To elicit SP, an LED spot was rotated about the stationary subjects while they tracked the spot with their eyes. Horizontal vestibulo-ocular reflex (VOR) was elicited by rotating an electrically powered turning chair in darkness. To test VOR suppression (VOR-S), the chair was rotated in fixed alignment with the LED spot being fixated by the subjects. Stimuli consisted of sinusoidal rotations (frequencies $0.1,0.2,0.4$, and 0.8 $\mathrm{Hz}$; peak displacement $\pm 16^{\circ}$; peak angular velocity $10,20,40$ and $80 \%$ s respectively). At each frequency, four to six stimulus periods were dispensed. The order of stimulus presentation was randomised.

Horizontal eye movements were recorded using an infrared light technique (Iris; Skalar, Delft, The Netherlands). Eye movement signals as well as stimulus position signals were sampled at $500 \mathrm{~Hz}$ and stored for off line analysis. Before and after each run, subjects performed saccades from the central fixation point towards lateral targets of $\pm 20^{\circ}$ amplitude for calibration.

\section{Magnetic resonance imaging}

MRI of the subjects' heads was performed using a Siemens vision scanner at $1.5 \mathrm{~T}$. The following sequences were obtained: (a) Tl weighted spin echo sequence (FLASH 3D, $\mathrm{TR}=40$ milliseconds, $\mathrm{TE}=6$ milliseconds, slice thickness $=1$ $\mathrm{mm}),(b) \mathrm{T} 2$ and proton weighted spin echo sequence

Abbreviations: SP, smooth pursuit eye movements; $M D$, myotonic dystrophy; CNS, central nervous system; MRI, magnetic resonance imaging; WML, white matter lesion; VOR, vestibulo-ocular reflex; VOR-S, suppression of VOR 
( $\mathrm{TR}=4440$ milliseconds, $\mathrm{TE}=119$ milliseconds, slice thickness $=2 \mathrm{~mm}$ ), and $(c)$ fluid attenuated inversion recovery (FLAIR) sequence $(\mathrm{TR}=9000$ milliseconds, $\mathrm{TE}=110$ milliseconds, $\mathrm{TI}=2261$ milliseconds, slice thickness $=4 \mathrm{~mm}$ ).

\section{Data analysis}

Saccades and artefacts were removed from the eye movement data with the help of an interactive computer program. The resulting smooth eye component and the stimulus signal that evoked the response were Fourier transformed and expressed in terms of gain and phase, separately for leftward and rightward movements. Gain was defined as the amplitude ratio of eye fundamental to stimulus fundamental (SP gain, VOR gain, VOR gain in the VOR-S condition (VOR-S gain)). These values were calculated for three or four cycles of the sinusoidal stimulation, taking the median value for statistical analysis.

WMLs were defined by an experienced neuroradiologist (independently, an experienced neurologist obtained similar results). Lesion locations were transferred to a brain scheme consisting of 10 horizontal templates $(\mathrm{zl}-\mathrm{zl} 10)$ in parallel with the bicommissural line. ${ }^{13}$ Each template consisted of two halves, each hemitemplate being subdivided into $6 \times 12$ pixels ( six columns in the lateral-medial direction, $\mathrm{xl}-\mathrm{x} 6 ; 12$ rows in the anterior-posterior direction, yl-yl2). A pixel was marked as lesioned if the WML filled more than $50 \%$ of the pixel. The transfer of MRI data to the templates followed anatomical landmarks (ventricle, major sulci, sylvian fissure, etc.). The procedure yielded 24 data sets ( 12 subjects, two hemispheres). We calculated the lesion probability for each pixel (number of lesions at a given pixel/24), thereby obtaining a topological map of lesion probabilities. Furthermore, the sum of lesion pixels per subject and hemisphere was related to the subject's ipsilateral SP gain at $0.8 \mathrm{~Hz}$ - that is, at the frequency with the highest demand on the pursuit system. In addition, SP gain at $0.8 \mathrm{~Hz}$ was assigned to each lesion pixel, separately for each subject and hemisphere (leftward pursuit, left hemisphere; rightward pursuit, right hemisphere). Mean SP gain for each lesion pixel was calculated across the 24 data sets and assigned to the corresponding pixel locations, yielding a topological SP gain map. Furthermore, each patient's SP gain at 0.8 $\mathrm{Hz}$ was transformed in terms of the mean and standard deviation of the control group (separately for rightward and leftward SP), such that

$$
Z=\left(\text { gain }_{\text {pat }}-\text { gain }_{\text {controlmean }}\right) / S D_{\text {control }}
$$

This $\mathrm{Z}$ value was then used to weight the effect of the WML, in analogy to the procedure for SP gain. To evaluate the effect of anterior versus posterior lesions, each hemisphere was further divided into an anterior (yl-y6) and posterior (y7-yl2) half. The average of pixel gains ( $\mathrm{SP}$ at $0.8 \mathrm{~Hz}$ ) in the anterior half was compared with that of the posterior half. Statistical significance was assessed by analysis of variance.

\section{RESULTS}

\section{Eye movements}

Figure 1A gives an example of SP in a patient with MD at 0.4 $\mathrm{Hz}$ stimulus frequency. The composite eye position corresponds to the target position, whereas the smooth eye component is considerably smaller, a fact that is compensated for by saccades.

In controls and patients, the gains in SP, VOR, and VOR-S for leftward and rightward eye movements were comparable $(\mathrm{p}>0.2)$.

\section{SP}

In normal subjects, SP gain remained almost constant at about unity up to $0.4 \mathrm{~Hz}$ and then decreased to a mean gain of $0.70(0.26)$ at $0.8 \mathrm{~Hz}$. The patients' gain was lower by about 0.1 at $0.1 \mathrm{~Hz}$, and decreased to $0.31(0.21)$ at $0.8 \mathrm{~Hz}$. Statistically,
A

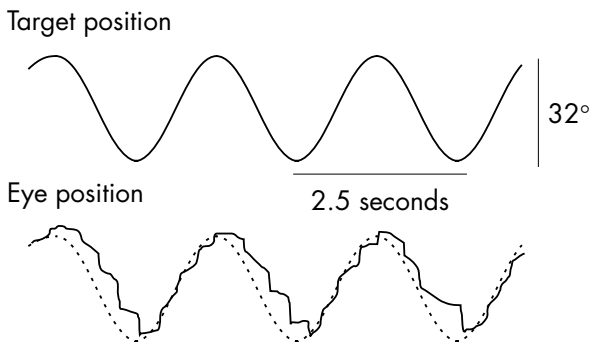

Smooth eye component

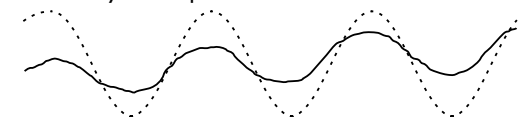

B

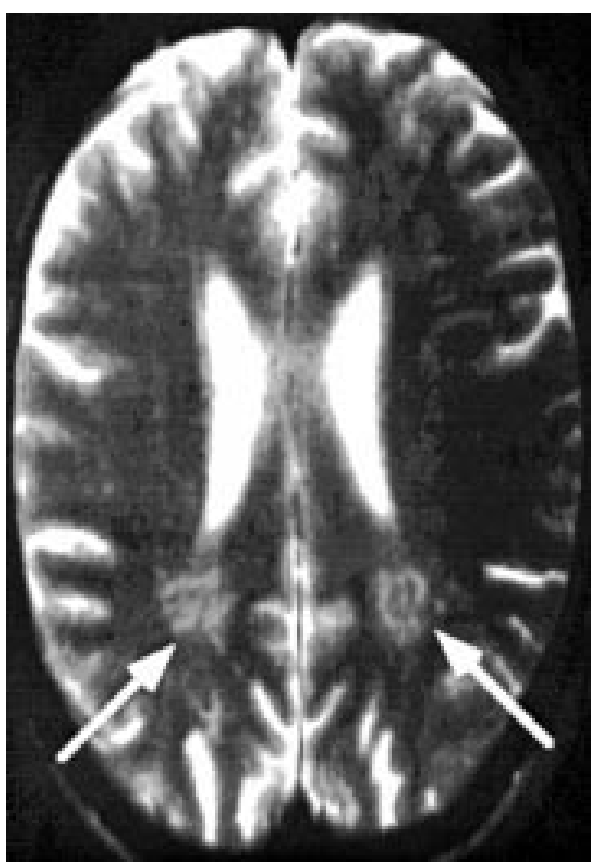

Figure 1 (A) Example of impaired smooth pursuit eye movement (SP) in a patient with myotonic dystrophy. The target moves sinusoidally at $0.4 \mathrm{~Hz}$ with a $32^{\circ}$ peak to peak amplitude (top trace). The eye position trace (middle) contains many saccades, indicating that the pursuit system is assisted by the saccadic system to keep the eye on the target. The smooth eye component (bottom trace) is obtained after removal of the saccades. The dotted trace gives "ideal" SP for comparison. (B) Horizontal magnetic resonance imaging scan (T2 weighted) of the same patient. Arrows indicate signal hyperdensities in the periventricular white matter around the occipital horn of the lateral ventricles.

we found significant effects for the two factors group and frequency (group: $F(1,176)=66.4 ; \mathrm{p}=0.0001$; frequency: $F(3,176)=59.0 ; \mathrm{p}=0.0001)$, as well as for the interaction between the two factors (difference between patients and controls increasing with frequency: $F(3,176)=5.16 ; \mathrm{p}=0.002)$. There was no correlation between SP gain at $0.8 \mathrm{~Hz}$ and disease severity in terms of the Karnofsky index $\left(r^{2}=0.1\right.$; $\mathrm{p}=0.6$ ) and between SP gain at $0.8 \mathrm{~Hz}$ and patients' age $\left(r^{2}=0.02 ; \mathrm{p}=0.7\right)$.

\section{VOR}

VOR gain increased slightly with increasing stimulus frequency reaching a mean of $0.69(0.23)$ at $0.8 \mathrm{HZ}$ in both patients and controls. There was no significant effect for the 
A

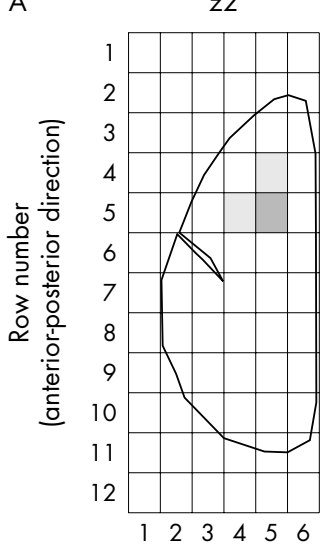

z3

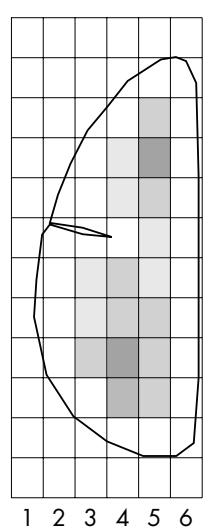

z4

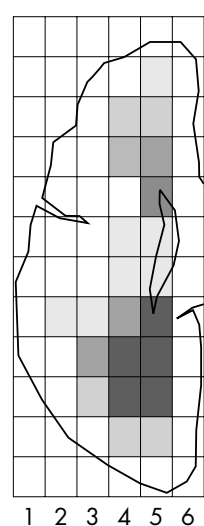

z5

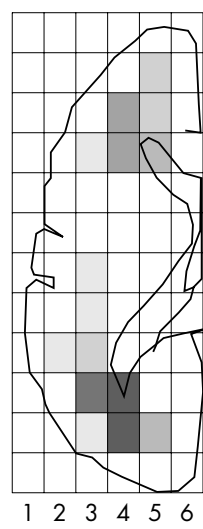

z6

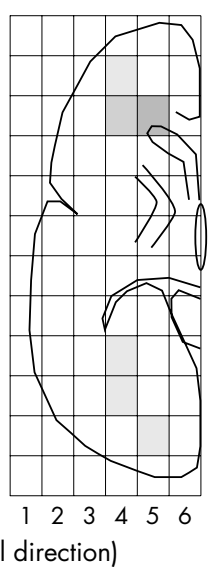

z7

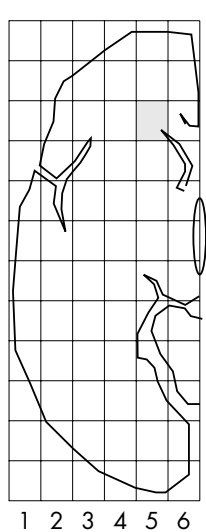

z8

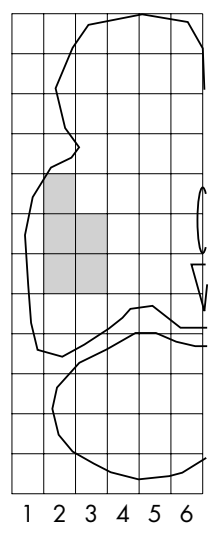

Column number (lateral-medial direction)

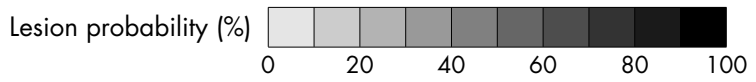

B

z2

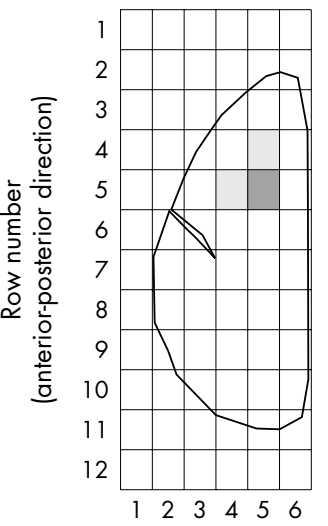

z3

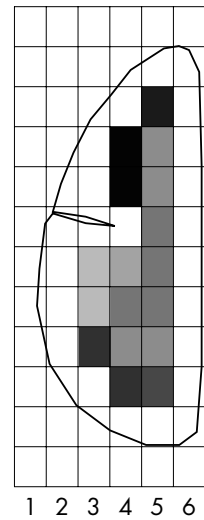

z4

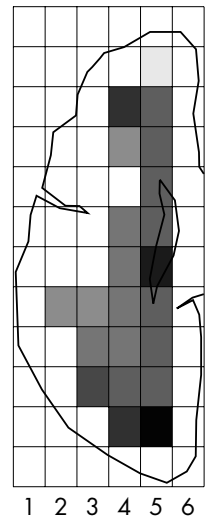

z5

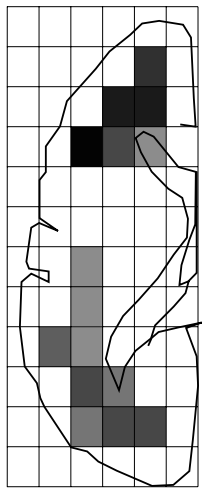

1233456

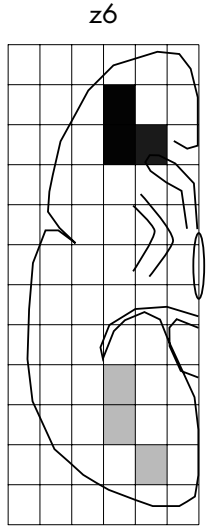

123456

Column number (lateral-medial direction)

Figure 2 (A) Lesion probability of each pixel in the standardised brain scheme. Lesions were found in 7 of 10 templates (labelled $z 2-z 8$ in the craniocaudal direction). Each template consists of $6 \times 12$ pixels (columns $\times 1-6$ in the lateral-medial direction, rows y $1-12$ in the anterior-posterior direction). Lesion probability (\%) is indicated by the grey level scale. Data for 24 hemispheres. Note the accumulation of lesions around the posterior and anterior lateral ventricle. (B) Effect of white matter lesions on smooth pursuit eye movements (SP), as weighted by the SP gain at $0.8 \mathrm{~Hz}$. Mean SP gain for each lesion pixel across 24 data sets is indicated by the grey level scale. Lowest SP gains are found in the anterior region, and a second broad zone with SP degradation is located around the occipital lateral ventricle.

factor group $(\mathrm{p}=0.4)$, but there was for the factor frequency $(F(3,173)=3.36 ; \mathrm{p}=0.02)$; interaction between the two factors was not significant $(\mathrm{p}=0.9)$.

\section{VOR-S}

VOR-S was incomplete in both groups. Mean VOR-S gain (indicating the remaining VOR gain) in controls was 0.07 $(0.05)$ at $0.1 \mathrm{~Hz}$ and increased slightly to $0.30(0.22)$ at $0.8 \mathrm{~Hz}$. The gain in patients with MD was higher by 0.04 at $0.1 \mathrm{~Hz}$ and increased to $0.37(0.16)$ at $0.8 \mathrm{~Hz}$. Statistical analysis showed significant effects for the factors group $(F(1,173)=11.4$; $\mathrm{p}=0.001)$ and frequency $(F(3,173)=60.3 ; \mathrm{p}=0.0001)$, but there was no significant interaction between these factors $(\mathrm{p}=0.8)$. There was no correlation between VOR-S gain at 0.8 $\mathrm{Hz}$ and disease severity in terms of the Karnofsky index $\left(r^{2}=0.02 ; \mathrm{p}=0.8\right)$.

\section{MRI}

Figure 1B gives an example of a T2 weighted horizontal MRI in the same patient as in fig lA. Note the periventricular hyperintensities around the occipital horn of the lateral ventricles (arrows).

Figure 2A shows the lesion probability of each pixel on a grey level scale for the 24 hemispheres investigated. The maximum of lesion probability is located near the posterior horn of the lateral ventricle within a broad lesion zone. A second, lower peak of lesion probability occurred around the anterior horn of the lateral ventricle within a smaller lesion zone. In two subjects, we found WMLs in the inferior temporal lobe. There was no correlation between the number of lesion pixels and a patient's age $\left(r^{2}=0.2 ; \mathrm{p}=0.2\right)$.

\section{SP and MRI}

A significant correlation between the extent of the lesionsthat is, the sum of lesion pixels per subject and hemisphereand ipsilateral SP gain at $0.8 \mathrm{~Hz}$ was not evident in our data set $\left(r^{2}=0.05 ; \mathrm{p}=0.3\right)$. In nine patients with $\mathrm{MD}$, we found anterior and posterior WMLs, and in three patients exclusively posterior WMLs. The latter showed a trend for somewhat 
higher SP gains than the former $(0.32 v 0.30$ with combined lesions). In fig $2 \mathrm{~B}, \mathrm{SP}$ gain at $0.8 \mathrm{~Hz}$ is assigned to the WML pixels and shown on a grey level scale. A broad peak of SP degradation (in terms of abnormally low gain) is seen around the occipital horn of the lateral ventricle. The maximum of SP degradation is located around the anterior horn. The statistical significance of this observation was tested by calculating the mean gain of all anterior (yl-y6) and posterior (y7-yl2) pixel gains across the 24 data sets; the mean gain of the anterior pixels amounted to 0.2 compared with 0.3 in the posterior pixels $(F(1,318)=5.23 ; \mathrm{p}=0.02)$. The $\mathrm{Z}$ score map (not shown) was almost identical with the SP gain map, reaching values of $\mathrm{Z}=2.5$ in anterior and $\mathrm{Z}=2.0$ in occipital WMLs.

\section{DISCUSSION}

Our study confirms a substantial SP deficit in patients with $\mathrm{MD}$, which was somewhat more pronounced in the present than in the previous study. We attribute this to the increasing stimulus velocity with increasing frequency in the present study. A parallel degradation of SP and VOR-S together with a normal VOR suggests a central rather than a peripheral origin of the SP deficit (VOR-S requires a "central" rather than a muscular effort). ${ }^{4} \mathrm{CNS}$ lesions in patients with MD have been reported previously ${ }^{9}{ }^{10}$ and were confirmed in terms of WMLs by MRI in our patients. WMLs showed two maxima, located around the anterior and occipital horn of the lateral ventricle. The lesions were associated with a major SP gain impairment at $0.8 \mathrm{~Hz}$ (confirmed by the $\mathrm{Z}$ score analysis), being more pronounced frontally than occipitally.

As to the neural substrate of the WMLs, it is known from monkey studies that fibres from the frontal eye fields descend to the dorsolateral pontine nuclei in the brainstem, passing through the anterior white matter. ${ }^{14}$ Fibres from the parietooccipital cortex (area MST in monkey) enter the internal sagittal stratum near the occipital horn of the lateral ventricle, also terminating in the dorsolateral pontine nuclei. ${ }^{15}$ In humans with circumscribed CNS lesions, ipsilateral SP impairment was found for target motion towards the side of the lesion, more commonly in patients with frontal eye field lesions than in patients with lesions in the posterior parietal cortex ${ }^{23}$ unless very extended lesions are considered. ${ }^{16}$ In all of our subjects, the WMLs were bilateral, almost symmetrical, and associated with an essentially symmetrical SP deficit. The most severe gain deficit (in terms of the largest difference between patients and controls) was found at the highest frequency/peak velocity tested $\left(0.8 \mathrm{~Hz} ; 80^{\circ} / \mathrm{s}\right)$. To allow a comparison with previous studies, we calculated gain values for 0.3 and $0.6 \mathrm{~Hz}$ from our frequency gain functions. The calculated values were comparable to those of Heide et $a l^{3}$ for controls and patients at $0.3 \mathrm{~Hz} / 30^{\circ} / \mathrm{s}$ (controls, 0.92 ; patients, 0.76 ), and somewhat lower than those of Morrow et al. ${ }^{2}$ Our observation of a gain degradation of sinusoidal SP, more with frontal than with parieto-occipital WMLs, complies with previous observations: while parieto-occipital regions play a major part in visual motion processing, the frontal eye fields appear to be involved in predictive and executive functions for SP at a stage downstream of parieto-occipital regions. ${ }^{37-19}$

\section{Authors' affiliations}

H Kimmig, M Petrick, T Mergner, Abteilung Neurologie,

Universitätsklinik Freiburg, Germany

M Orszagh, Sektion Neuroradiologie, Universitätsklinik Freiburg

Correspondence to: Dr Kimmig, Neurologische Universitätsklinik Freiburg, Breisacher Str 64, D-79106 Freiburg, Germany; kimmig@uni-freiburg.de

Received 4 September 200

In revised form 8 January 2002

Accepted 8 February 2002

\section{REFERENCES}

1 Leigh RJ, Zee DS. The neurology of eye movements. New York: Oxford University Press, 1999

2 Morrow MJ, Sharpe JA. Cerebral hemispheric localization of smooth pursuit asymmetry. Neurology 1990:40:284-92.

3 Heide W, Kurzidim K, Kompf D. Deficits of smooth pursuit eye movements after frontal and parietal lesions. Brain 1996;1 19:1951-69.

4 Anastasopoulos D, Kimmig H, Mergner T, et al. Abnormalities of ocular motility in myotonic dystrophy. Brain 1996;119:1923-32.

5 Bollen E, den Heyer JC, Tolsma MH, et al. Eye movements in myotonic dystrophy. Brain 1992;1 15:445-50.

6 Verhagen WI, ter Bruggen JP, Huygen PL. Oculomotor, auditory, and vestibular responses in myotonic dystrophy. Arch Neurol 1992:49:954-60.

7 Shaunak S, Orrell R, Henderson L, et al. Saccades and smooth pursuit in myotonic dystrophy. J Neurol 1999;246:600-6.

8 Anastasopoulos D, Kimmig H, Mergner T. Abnormalities of ocular motility in myotonic dystrophy. Brain 1997; 120:1908-9.

9 Glantz RH, Wright RB, Huckman MS, et al. Central nervous system magnetic resonance imaging findings in myotonic dystrophy. Arch Neurol 1988;45:36-7.

10 Damian MS, Bachmann G, Herrmann D, et al. Magnetic resonance imaging of muscle and brain in myotonic dystrophy. J Neurol 1993;240:8-12.

11 Brook JD, McCurrach ME, Harley HG, et al. Molecular basis of myotonic dystrophy: expansion of a trinucleotide (CTG) repeat at the $3^{\prime}$ end of a transcript encoding a protein kinase family member. Cell 1992;68:799-808.

12 Karnofsky DA, Burchenal JH, Armistead GC, et al. Triethylene melamine in the treatment of neoplastic disease. Arch Int Med 1951:87:477-516.

13 Talairach J, Tournoux P. Co-planar stereotaxic atlas of the human brain. Stuttgart: Thieme, 1988

14 Leichnetz GR. Inferior frontal eye field projections to the pursuit-related dorsolateral pontine nucleus and middle temporal area (MT) in the monkey. Vis Neurosci 1989:3:171-80.

15 Tusa RJ, Ungerleider LG. Fiber pathways of cortical areas mediating smooth pursuit eye movements in monkeys. Ann Neurol 1988;23:174-83.

16 Lekwuwa GU, Barnes GR. Cerebral control of eye movements. I. The relationship between cerebral lesion sites and smooth pursuit deficits. Brain 1996;1 19:473-90.

17 MacAvoy MG, Gottlieb JP, Bruce CJ. Smooth-pursuit eye movement representation in the primate frontal eye field. Cereb Cortex $1991 ; 1: 95-102$.

18 Keating EG. Frontal eye field lesions impair predictive and visually-guided pursuit eye movements. Exp Brain Res 1991;86:311-23.

19 Keating EG. Lesions of the frontal eye field impair pursuit eye movements, but preserve the predictions driving them. Behav Brain Res 1993;53:91-104. 